\title{
The Wide Field Cameras onboard the BeppoSAX X-ray Astronomy Satellite
}

\author{
R. Jager, W.A. Mels, A.C. Brinkman, M.Y. Galama, H. Goulooze, J. Heise, P. Lowes, J.M. Muller, \\ A. Naber, A. Rook, R. Schuurhof, J.J. Schuurmans, and G. Wiersma \\ Space Research Organisation Netherlands, Sorbonnelaan 2, 3584 CA Utrecht, The Netherlands
}

Received November 26, 1996; accepted January 29, 1997

\begin{abstract}
The two Wide Field Cameras (WFCs) currently flying on the Italian/Dutch X-ray satellite BeppoSAX, image the $\mathrm{X}$-ray sky in the energy range of $1.8-28 \mathrm{keV}$. The field of view is $20^{\circ}$, the angular resolution is 5 arcmin and the energy resolution is $20 \%$ at $6 \mathrm{keV}$, while the source location accuracy will generally be better than one arcmin. All values are at Full Width Half Maximum. The design is based on the coded mask principle where mask and detector both have sizes of about $25.6 \times 25.6 \mathrm{~cm}^{2}$. The detector is a Multi Wire Proportional Counter with a position resolution which is better than $0.5 \mathrm{~mm}$ for energies below $10 \mathrm{keV}$. The mask pattern is based on a so called triadic residue set with elements of $1 \mathrm{~mm}^{2}$ of which $33 \%$ are transparent for X-rays. The limiting sensitivity of the WFCs is a few mCrab in $10^{5} \mathrm{~s}$. The design and performance of the instrument is described here along with some calibration results and the principle of the image reconstruction.

The WFCs represent the latest generation of coded mask X-ray cameras. Their unrivalled field of view, combined with appropriate spatial and temporal resolution and modest energy resolution make the instruments very well suited to perform extensive studies of large areas of the sky and to study transient X-ray phenomena.
\end{abstract}

Key words: instrumentation: detectors - methods: data analysis - methods: observational - X-rays: general

\section{Introduction}

BeppoSAX is a scientific X-ray satellite programme developed by the Italian Space Agency (ASI) with a contribution from the Netherlands agency for Aerospace Programs (NIVR) and the Space Research Organisation Netherlands (SRON) (Boella et al. 1996a). Galactic and extragalactic sources will be observed in the energy band

Send offprint requests to: R. Jager: r.jager@sron.ruu.nl of $0.1-300 \mathrm{keV}$. The satellite has been launched on April 30, 1996 and operates succesfully in a low equatorial orbit. SAX carries six scientific instruments, including two identical Wide Field Cameras (WFCs), pointing $180^{\circ}$ away from each other, perpendicular to the sun vector and to the other instruments (Parmar et al. 1996; Boella et al. 1996b; Manzo et al. 1996; Frontera et al. 1996).

The WFCs are coded mask cameras for imaging of hard X-ray sources and are part of the Dutch contribution to BeppoSAX. Three WFCs have been developed and built by SRON Utrecht, two of which were integrated in the satellite and one was used for testing purposes and as a flight spare instrument.

Other comparable coded mask type cameras that have flown or will fly are a.o. the SL2-XRT experiment onboard Spacelab-2 (Willmore et al. 1985), COMIS/TTM on KVANT/MIR (Brinkman et al. 1983), ART-P on the GRANAT mission (Sunyaev et al. 1990) and MART/LIME on Spectrum X-Gamma (Bazzano et al. 1994). An overview of X-ray astronomy missions is given by Bradt et al. (1992). The WFCs can carry out spatially-resolved simultaneous monitoring of compact X-ray sources in crowded fields with high sensitivity. This allows for systematic spectral variability studies of different types of compact X-ray sources over a large range of timescales. Surveys of selected sky regions such as the galactic centre are carried out to search for and study the characteristics of X-ray transients. The WFCs image $\mathrm{X}$-ray emission of various classes of astrophysical objects, ranging from nearby stars to active galactic nuclei. In addition, the WFCs will monitor large regions of the sky for previously unknown X-ray sources, such as black hole candidates and neutron stars, for follow-up studies with the narrow field instruments onboard BeppoSAX.

Here we discuss the design and performance of the instrument as well as the principle for the image reconstruction and the analysis software. 


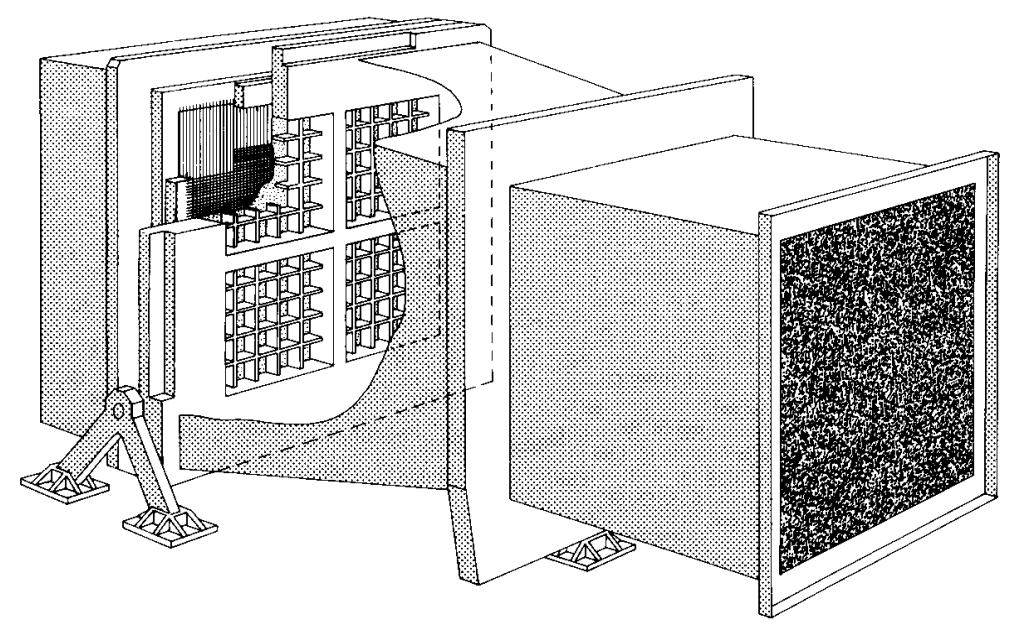

Fig. 1. WFC instrument schematic drawing. A coded mask is placed $70 \mathrm{~cm}$ in front of a two dimensional position and energy sensitive detector. The total mass is $42.5 \mathrm{~kg}$ and the power consumption is $14.1 \mathrm{~W}$

\section{WFC description}

\subsection{Overall instrument}

The principle of the WFC is the shadow mask camera (Dicke). A two-dimensional position- and energy sensitive detector (Multi Wire Proportional Counter) is placed behind an opaque screen with a pseudo random array of holes, a so-called shadow mask or coded mask. X-ray sources in the field of view cast shadows of the mask on the detector, each displaced according to the position of the source. A cross-correlation (Fenimore et al. 1978) carried out on ground, reconstructs the position and flux of all sources in the observed part of the sky. The WFC has a high sensitivity over a large field of view and at the same time a good angular resolution over the whole field (due to small mask holes). Figure 1 shows a schematic drawing of the WFC.

The detector and the mask have sizes of $25.5 \times 25.5 \mathrm{~cm}^{2}$ and $25.6 \times 25.6 \mathrm{~cm}^{2}$, respectively. A stainless steel structure between detector and mask allows the X-ray photons to reach the detector through the mask only and fixes the position of the mask $0.7 \mathrm{~m}$ away from the detector. In front of the mask a $6 \mu \mathrm{m}$ thick kapton foil is placed as a thermal shield. The field of view is $20^{\circ}$ square (FWHM) although sources can be detected with less sensitivity in a field of $40^{\circ} \times 40^{\circ}$ in total. The angular resolving power is 5 arcmin (FWHM), determined by the mask-to-detector distance, the size of the mask elements which is $1 \times 1 \mathrm{~mm}^{2}$ and the detector position resolution. The energy resolution is $20 \%$ at $6 \mathrm{keV}$ and $22 \%$ at $22 \mathrm{keV}$. The source location accuracy is better than one arcminute, limited mainly by
Table 1. WFC overall characteristics

\begin{tabular}{ll}
\hline Parameter & Value \\
\hline Energy range & $1.8-28 \mathrm{keV}$ \\
Energy resolution at $6 \mathrm{keV}$ & $20 \%$ over full area \\
Effective area & $140 \mathrm{~cm}^{2}$ \\
Field of view & $20^{\circ} \times 20^{\circ}(\mathrm{FWHM})$ \\
Angular resolving power & $5 \mathrm{arcmin}$ \\
Source location accuracy & $<1$ arcmin \\
Sensitivity in $10^{5} \mathrm{~s}$ & few $\mathrm{mCrab}$ \\
Mass & $42.5 \mathrm{~kg}$ \\
Power consumption & $14.1 \mathrm{~W}$ \\
Maximum countrate & $2000 \mathrm{c} \mathrm{s}^{-1}$ \\
$\left(\right.$ Crab $\left.=270 \mathrm{c} \mathrm{s}^{-1}\right)$ & \\
\hline
\end{tabular}

the satellite pointing stability and the statistical noise of the observed sources.

The limiting sensitivity is a few mCrab in $10^{5} \mathrm{~s}$ but varies with the background X-ray flux and thus with the pointing direction in the sky. At high galactic latitudes the background will be dominated by the diffuse X-ray background (130 $\mathrm{c} \mathrm{s}^{-1}$ ) plus the detector background $\left(20 \mathrm{c} \mathrm{s}^{-1}\right)$ while near the galactic plane the main contribution is from strong X-ray sources. Figures 2 and 3 show the minimum detectable source strength and the time needed to detect time variability. From early measurements it could be shown that for reliable source detection a confidence level higher than $5 \sigma$ is needed. Detailed in-flight calibrations will clarify this issue. 


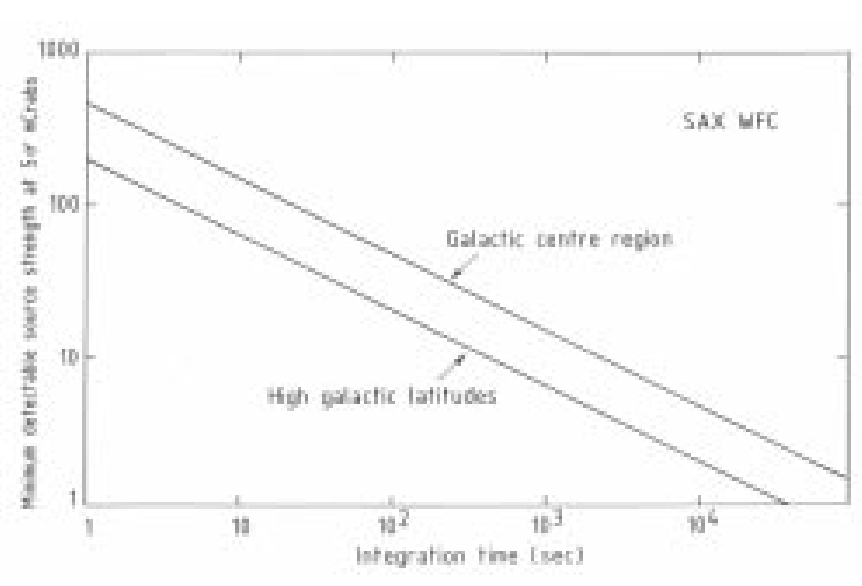

Fig. 2. Minimum detectable source strength for two sky regions. The sensitivity is on average higher at high galactic latitudes where the overall flux from X-ray sources is less

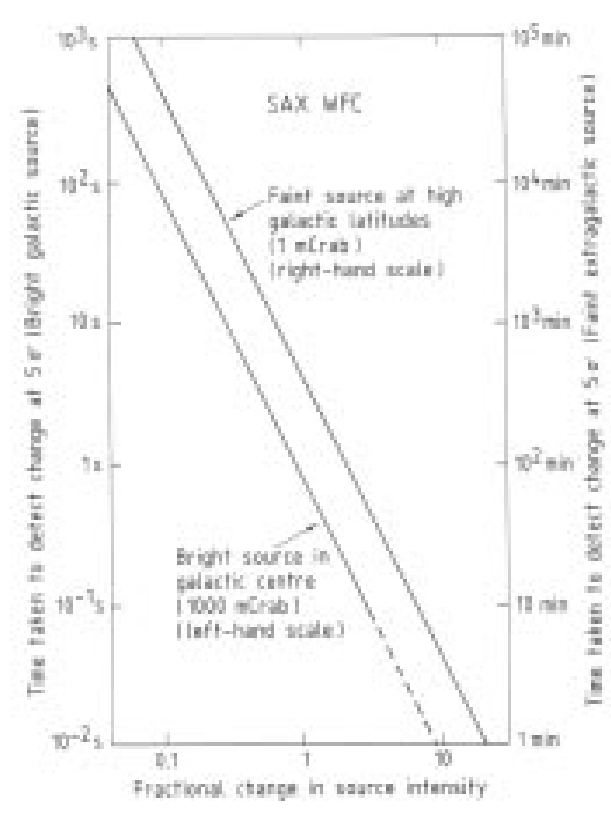

Fig. 3. Observing time required to detect source variability for two sky regions, in less crowded regions the sensitivity for source variability is higher

The WFCs can be used in two scientific and three diagnostic modes. The scientific modes are the Normal (imaging) Mode (NM) and the High Time Resolution Mode (HTRM). The diagnostics modes are DM1, DM2 and the Background Analysis Mode (BAM).

In NM the time resolution is $0.5 \mathrm{~ms}$ for every block of 4 photon events. Each event is labelled with 26 bits, 10 bits each for $x$ - and $y$-position, 1 bit for indicating the upper or lower detector half and 5 energy bits. The maximum event processing rate is $2000 \mathrm{~s}^{-1}$, limited by the instrument processor performance and the satellite telemetry rate.

In HTRM the time resolution is $0.25 \mathrm{~ms}$ per event while the maximum processing eventrate is $5000 \mathrm{~s}^{-1}$. In this mode no event position information can be produced, this mode is useful for timing analysis of strong sources.

For diagnostic purposes two Diagnostic Modes (DM1 and DM2) are available. In these modes no onboard processing takes place and all raw event data is available. For background calibration purposes a Background Analysis Mode (BAM) is implemented.

When the available telemetry in NM for the WFCs is not sufficient, the instruments can be programmed to reduce their telemetry. Processing of every $n$-th event will then be stopped at the input, where $n$ is an integer between 2 and 4 . If this reduction is not sufficient and the telemetry buffer becomes full, no more events will be processed until the buffer is read out.

\subsubsection{Design considerations}

In the WFC, coded mask and detector have the same size; this corresponds to the so-called "simple" camera described by Sims 1980. This design does not allow complete coding, in contrast to the "optimum" camera (Proctor et al. 1979), where mask and detector do not have the same size. However, the "simple" camera has the following significant advantages over the "optimum" camera:

1. The angular resolution is twice as good, the Field of View (FOV), the sizes of mask and detector elements and the mask to detector distance being the same;

2. For off-axis positions the noise from the diffuse background per reconstructed sky element will be lower;

3. Images of sources in the FOV which are more than half a FOV apart (i.e. more than $20^{\circ}$ ), do not mutually disturb each other by Poisson noise or mask noise. This is due to the fact that they do not produce overlapping shadow patterns on the detector. For an "optimum" camera with a mask larger than the detector, all sources in the FOV always overlap.

These arguments plus the fact that the "simple" camera was physically easier to accomodate in BeppoSAX have led to the choice of the present design.

\subsection{Detector}

The detector is an improved less heavy version of the COMIS (Coded Mask Imaging Spectrometer) detector. COMIS/TTM (Brinkman et al. 1983) is a coded mask camera instrument in the KVANT module of the MIR space station and is succesfully operating since 1988. 


\subsubsection{Overall configuration}

The detector configuration is a conventional two dimensional position sensitive Multi Wire Proportional Counter (Charpak 1985). The detector consists of two separate counters in one sealed housing: the main photon counter and the guard counter used for background rejection, see Fig. 4.

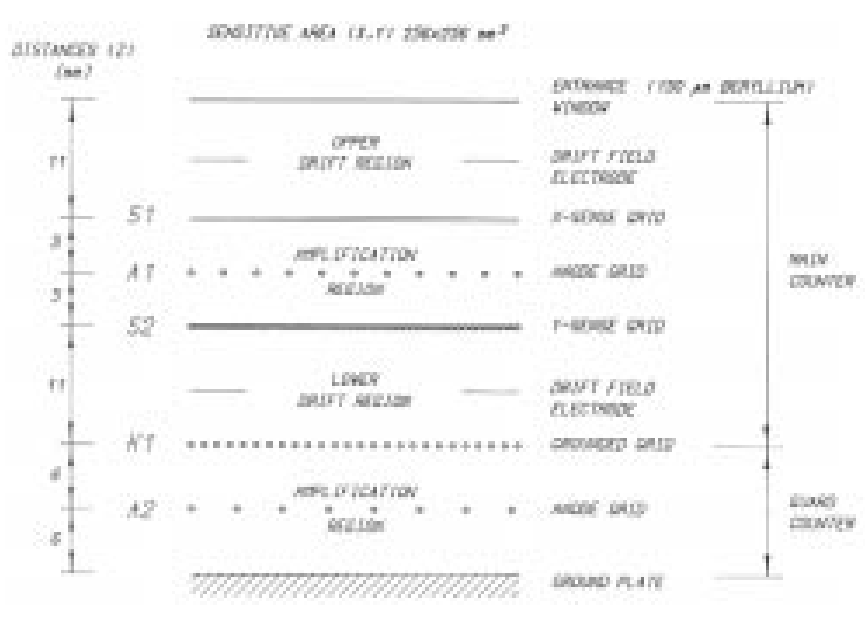

Fig. 4. Schematic diagram of the WFC detector showing the configuration of the main and the guard counter. The guard counter serves to reject background and spurious events

Both counters are separated by a grounded wiregrid K1. The detector contains drift field electrodes in both drift regions, and wire grids in the amplification regions.

Because of weight constraints, the detector housing is made of titanium with a beryllium entrance window support structure (Fig. 5). This structure was built by Electro Fusion Corporation of Fremont, U.S.A.

A $1 \mathrm{~mm}$ aluminium shield covers the titanium walls at the outside of the housing to minimise the AC magnetic field susceptibility of the detector. The detector is sealed with an indium gasket. The size of the detector entrance window is $255 \times 255 \mathrm{~mm}^{2}$. The actual window consists of a $150 \mu \mathrm{m}$ beryllium foil epoxied to the beryllium window support structure. The support structure subdivides the window into $10 \times 10$ subwindows). The total detector sensitive area is $520 \mathrm{~cm}^{2}$.

\subsubsection{The grid system}

Figure 4 shows the lay-out of the grid system, which consists of five wire grids. These grids serve as anodes, sense grids, and electrical grounding (grids A1 and A2, S1 and $\mathrm{S} 2$, and $\mathrm{K} 1$, respectively). The wire grids are mounted with respect to each other with an accuracy of about $40 \mu \mathrm{m}$. Table 2 gives dimensions and voltages for the gridwires. In general, the wires of a grid are interconnected.
We now discuss the individual grids in more detail, starting with the anode grids.

The anode grid A1 is located half way down the Main Counter. It serves to obtain energy information on the impingent X-ray photons, and triggers the event processing. Three wires are not connected electrically to this grid, they function as the anode of a separate so-called Wall Counter.

Likewise, the Guard Counter possesses an anode grid A2. We note that the wires at the edges of both A1 and A2 have increased diameters. This lowers the local electric field, and therefore prevents high voltage break-down.

The sense grids S1 and S2 allow the position of the photon track to be determined in the $x$ - respectively the $y$-direction. To this aim, they are positioned at both sides of the anode grid A1 of the Main Counter, while running perpendicular and parallel, respectively, to the anode wires of A1. A special interconnection scheme, using fine and coarse sections, serves to read the pulses from the sense grids, as shown in Fig. 6. In each sense grid, the wires are grouped into 48 fine sections. Four fine sections in turn make up one coarse section, so that there are 12 coarse sections in total. Every fine section is connected to the corresponding location within a coarse section.

All grids together have 35 signal outputs. The wires themselves are composed of gold plated tungsten and fixed with an accuracy of $10 \mu \mathrm{m}$ on ceramic macor strips with glue spots. The interconnection patterns for the fine and coarse sections have been deposited using thick-film techniques, the wires being connected with conductive epoxy.

The wire grid system $\left(275 \times 275 \mathrm{~mm}^{2}\right)$ has been chosen larger than the entrance window to minimise distorting boundary effects on the spatial and spectral response. In this way, secondary radiation originating from the housing walls and grid supports can be discriminated against. Furthermore, a field correction plate was installed at both sides of the grid system. These plates guide the field lines in both drift regions (see Fig. 4) the direction perpendicular to the entrance window.

Table 2. Wire dimensions and voltages of the detector grid system.

\begin{tabular}{llllll}
\hline Parameter & & S1/S2 & A1 & A2 & K1 \\
\hline wire diameter & {$[\mu \mathrm{m}]$} & 50 & 20 & 20 & 50 \\
wire pitch & {$[\mathrm{mm}]$} & 0.6 & 3.0 & 0.6 & 1.2 \\
wire voltage & {$[\mathrm{V}]$} & 900 & 3750 & 2700 & 0 \\
\hline
\end{tabular}

\subsubsection{Gas filling}

The detector is filled at 2.2 bar with a mixture of $\mathrm{Xe}$ $(94 \%), \mathrm{CO}_{2}(5 \%)$ and $\mathrm{He}(1 \%)$. While the gas gain can be 


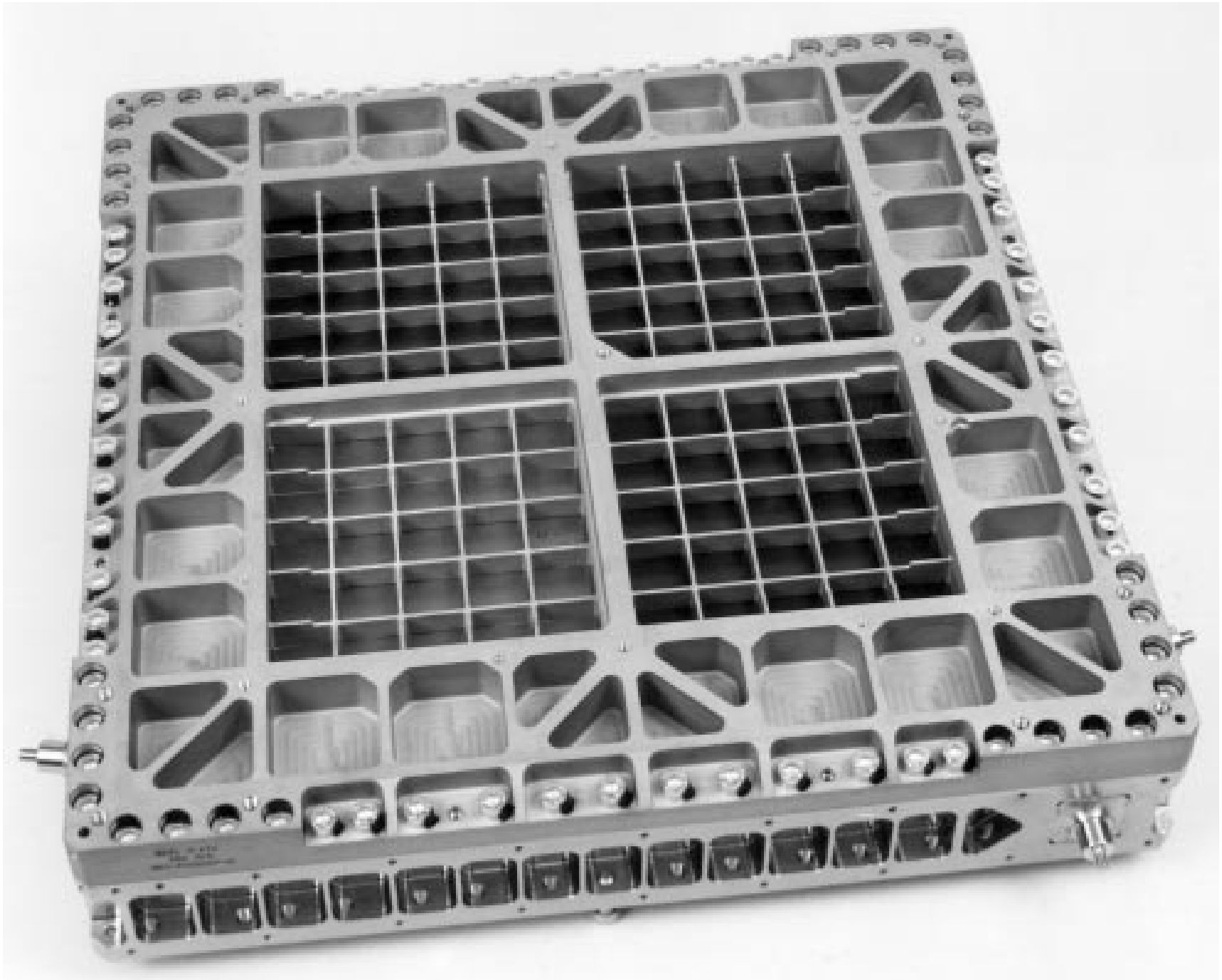

Fig. 5. Front view of the WFC detector, clearly visible is the beryllium window support structure. The detector housing is made of titanium. The size is $40 \mathrm{~cm} \times 40 \mathrm{~cm}$

adjusted by changing the high voltage supply, the nominal gain value is kept at 15000 .

\subsubsection{Inflight calibration sources}

In order to continuously verify the energy and spatial response, a number of inflight calibration sources are mounted to the detector window support structure. Nine "pinhole" source containers containing ${ }^{55} \mathrm{Fe}$ radio-active material are used to monitor the spatial response of the detector. Each pinhole source emits $6 \mathrm{keV}$ photons in a beam with a diameter of $<0.2 \mathrm{~mm}$ FWHM and with an intensity of about 0.01 photon per second. Eight sources are located at the borders of the sensitive area, while one source is in the centre of the sensitive area. All sources are at positions where the spatial response is most sensi- tive to minute displacements of the individual grids and to electrical interference.

A "cocktail source" holder with ${ }^{55} \mathrm{Fe}$ and ${ }^{109} \mathrm{Cd}$ radio active material emits a mixture of $6 \mathrm{keV}$ photons $\left(10 \mathrm{c} \mathrm{s}^{-1}\right)$ and $22 \mathrm{keV}$ photons $\left(1 \mathrm{c} \mathrm{s}^{-1}\right)$ respectively in a beam of $1 \mathrm{~mm}$ FWHM. This source is used to verify the energy response and the short term stability of the gas gain.

\subsubsection{Design considerations}

The following properties are desirable for an optimum detector:

- an extended energy range

- a large effective area, and 


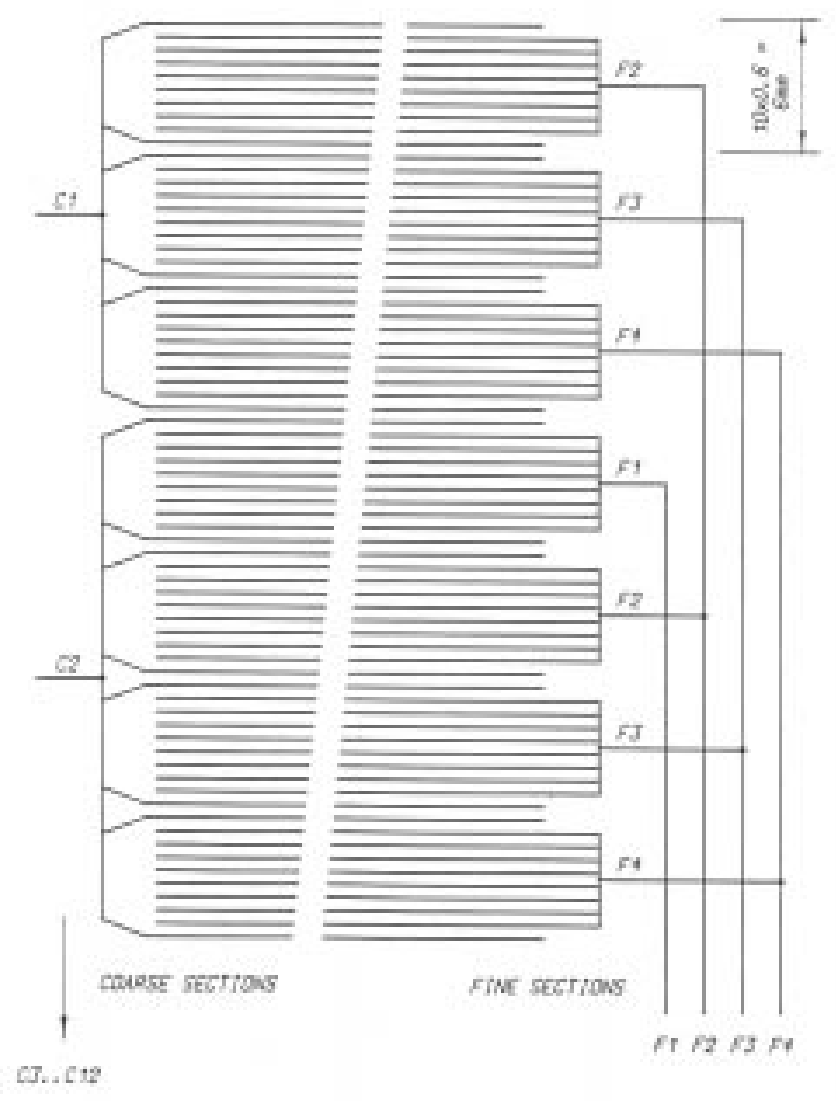

Fig. 6. Interconnection diagram for the wires of the sense grids S1 and S2 (see Fig. 4). The position readout scheme for one of the two detector coordinates is shown. The 12 coarse sections C1..C12 each contain 8 wires while the 4 fine sections F1..F4 each consist of 96 wires

- high spatial resolution, both for on-axis and inclined beams.

However, the corresponding requirements are in part contradictory so that a balance must be found. In these considerations should be included:

- the gaspressure,

- the thickness of the entrance window foil,

- height and pitch of the bars of the window support structure, and

- the overall detector mass.

We now explain the necessary trade-offs between these parameters.

\section{- Extended energy range.}

To detect high energy photons, the gas density i.e. pressure has to be sufficiently high. However, to allow low energy photons to enter the detector, the entrance window foil has to be thin. This implies that the sub windows of the window support structure must be small and the bars high to prevent the window foil from bursting and the whole support structure from bending. Bending of the structure and foil causes non linearity in the spatial response.

- Large effective area for inclined beams.

A large effective area can be achieved by choosing a "transparent" window support structure, implying small support structure sub windows and low bars.

- Good spatial resolution for inclined beams.

Optimal imaging capabilities have to be achieved over the whole Field of View and the whole energy range. High energy photons, however, penetrate the gas considerably before they are absorbed. This causes a tail on the Point Spread Function for photons arriving from the edge of the FOV. To minimize this effect, the gas pressure must be sufficiently high. Again this implies small sub windows and high support bars.

To keep the mass down, the detector housing is of titanium while the window support structure is entirely made of beryllium. The gas pressure was chosen to be 2.2 bar. This allows an entrance foil thickness of $150 \mu \mathrm{m}$ and a sub window area of $23 \times 23 \mathrm{~mm}^{2}$. The width and height of the support bars was chosen to be $1.5 \mathrm{~mm}$ and $10 \mathrm{~mm}$, respectively. To increase rigidity the window support central cross bars are made $22 \mathrm{~mm}$ high and $13 \mathrm{~mm}$ wide.

The spatial resolution of the photon detection is determined by the gas filling, the gas gain, the grid system and the electronic noise. This has been discussed in an earlier paper on the COMIS detector (Mels et al. 1988).

Photon detection in the amplification regions results in larger pulse heights than in the drift regions. In the upper part of the energy range the energy resolution is easily degraded by this effect. This is not caused by electron attachment but arises because the gain is affected by space charge in the avalanche (Mels et al. 1988). The charge density in this avalanche is higher for drift region events than for amplification region events due to the binning effect of the sense wires. This difference becomes smaller when the electric field in the drift region is increased. A drift voltage of $900 \mathrm{~V}$ was needed to obtain a resolution of $19 \%$ for a collimated beam of $22 \mathrm{keV}$ photons.

\subsection{Electronics}

\subsubsection{Readout electronics}

There are 35 detector output signals, each of which is connected to its own readout channel. The electronics of such a detector readout channel consist of a charge sensitive preamplifier followed by a pulse shaping circuit.

The anode channels have fast pre-trigger outputs. The triggers are used to initiate an event handling cycle as described below in Sect. 2.3.2. They trigger the pile-up sensing circuit and serve for anti-coincidence purposes.

Each coarse section channel of the sense grids has a trigger output to set the corresponding bit in two 12-bits coarse address registers. The pulse height of the fine section signals and the anode A1 signal are digitised by a 
12-bit ADC. These channels are equipped with a baseline restorer to decrease the sensitivity to microphonics and pile-up effects. The charge collection efficiency in these channels is about $40 \%$.

The event selection and position computation is performed by the front end electronics and the event processor in real time. For each validated event a data packet is generated. The data is then formatted in blocks of fixed length by the communication processor and stored in the instrument memory. The data handling system reads the memory and stores its contents on the onboard tape recorder. Rate meter time profiles are included to enable monitoring of the count rates on the anode grids and other trigger outputs. With this data the following diagnostic parameters can be calculated: the instrument dead time, the high energy $(>30 \mathrm{keV})$ event rate, the background event rate and the rate of pile-up events.

Once per orbit the contents of the tape recorder is transmitted to the ground station. In case modified procedures of onboard data handling are required, all the software and look-up tables can be updated by uplink from the ground station.

\subsubsection{Event handling}

By definition an event occurs in the main counter if either a photon or a particle ionises the gas in the chamber. The resulting cloud of electrons drifts through the sense grid to the amplification region. In case of detection of a photon with an energy below the $35 \mathrm{keV} \mathrm{Xe} K$-absorption edge, an avalanche occurs around one single anode wire or two neighbouring wires. If the charge impulse on the anode is sufficiently high, the event handling cycle is initiated.

First the frontend electronics read the detector signals. Subsequently the pulse height in the eight fine section and the main anode channel is stored in analog stretchers. The pattern of the triggered anode channels and the coarse section channels is latched into registers. At the end of this phase the event handling cycle is aborted if one or more veto conditions are satisfied. Veto conditions are:

- coincident event in guard and/or wall counter,

- illegal pattern of triggered coarse section channels,

- a pile-up condition when the time interval between present and previous event is too short.

The first two conditions discriminate between photons and particles.

If an event is accepted by the front end electronics, the analog signals stored in the stretchers are digitised and the event position is determined. By comparing the charge induced on sensegrids S1 and S2, the event processor also determines whether the event occurred above or below the anode grid A1. This information is useful to improve the position resolution at higher energies for inclined beams, and also serves to reject spurious low energy events $(<10 \mathrm{keV})$ in the lower drift region.
The point of absorption of a photon in lateral direction is derived from the centre of gravity $Z$ of the charge distribution induced by the avalanche charge on the sense grids. The coarse section index is determined from the pattern of triggered coarse section channels. The position of $Z$ within that coarse section is calculated using a simple linear combination of the charge values measured on the fine sections. The resulting value for the "position estimator" has a non-linear relationship with the photon position. This is corrected for by look-up tables inflight.

\subsection{Mask}

The mask consists of a $0.1 \mathrm{~mm}$ thick stainless steel plate with a $2 \mu \mathrm{m}$ gold coating on both sides. It contains a pattern of $256 \times 256$ mask elements of $1 \times 1 \mathrm{~mm}^{2}$. Of the mask elements $33 \%$ are perforated, i.e. transparent to $\mathrm{X}$-rays. The mask has been produced by means of an etching process. To assure the structural integrity of the mask, the size of the open elements is $0.9 \times 0.9 \mathrm{~mm}^{2}$. The mask hole pattern is based on a so-called "triadiç residue (in 't Zand et al. 1994) set and is shown in Fig. 7.

\subsubsection{Design considerations}

The arguments behind the choice of the coded mask parameters were presented by in 't Zand et al. (1994) and are summarized here.

The optimum open fraction of a coded aperture depends on the spatial response of the instrument. For the BeppoSAX WFCs the optimum open fraction lies between 0.25 and 0.33 . The requirement of a high sensitivity to X-ray bursts then led to the choice of 0.33 for the mask transparency. Compared with a more traditional choice of 0.50 , not only the sensitivity improves but also the telemetry that is needed decreases with one-third.

A transparency of 0.33 can not be achieved with Uniform Redundant Array (URA) patterns which are based on quadratic or bi-quadratic residue sets. By extrapolation of these sets we have arrived at a "triadiç residue set, where the non-zero shift autocorrelation is 3-valued with 2 values being the same.

A URA pattern in an "optimum" design (see Sect. 2.1.1) would have a 1-valued autocorrelation (i.e. flat sidelobes). In our camera design these autocorrelation features can however be permitted. Due to the shadowing effect of both the collimator and the detector window support structure, the coding of the image is never complete. The resulting coding noise can be largely removed during the image reconstruction process, see Sect. 4.2.

\section{Instrument performance}

\subsection{Introduction}

In Table 3 a summary of the performance characteristics is presented. Most data derive from measurements on a 


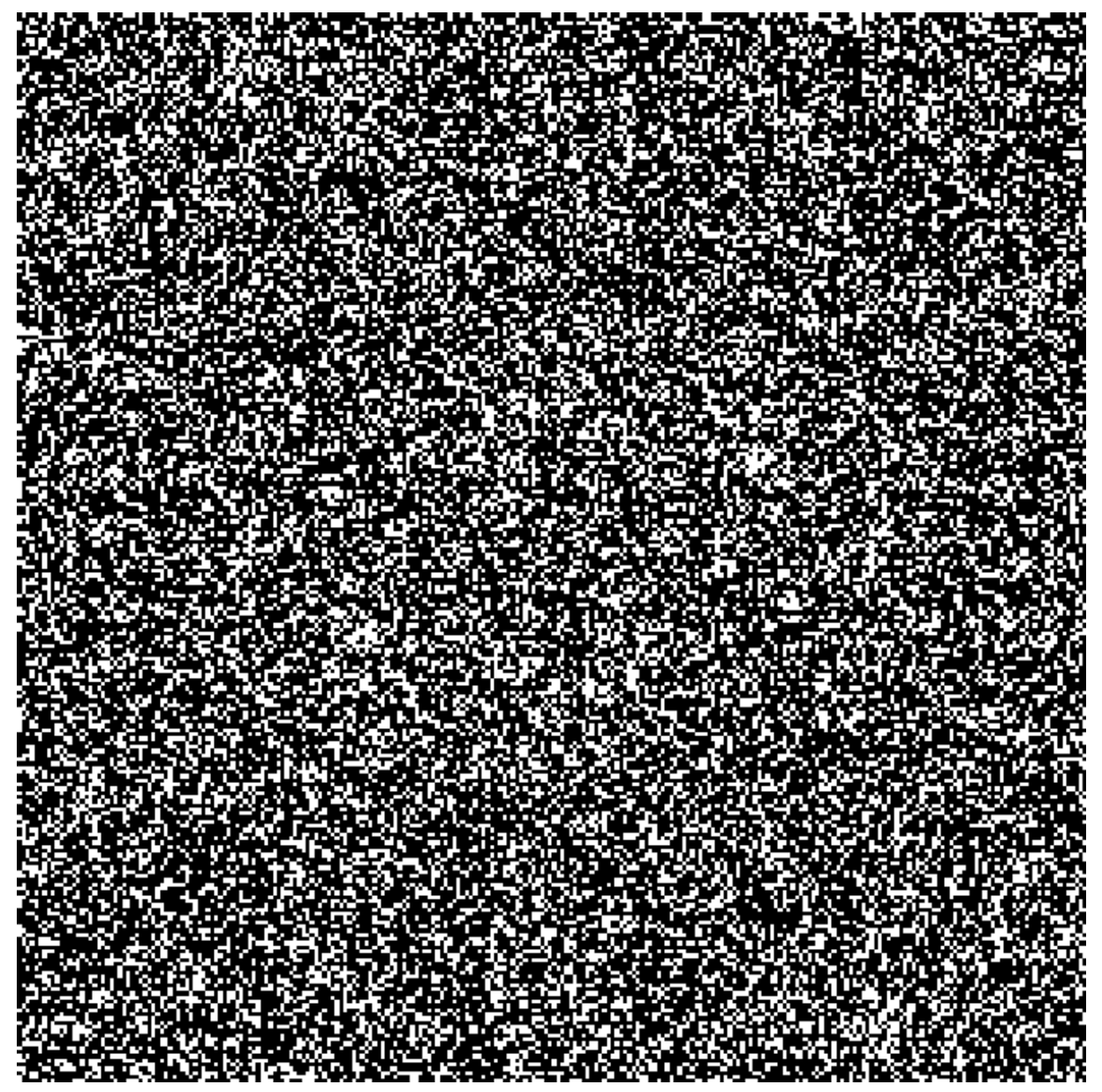

Fig. 7. WFC coded mask pattern consisting of $256 \times 256$ elements of $1 \times 1 \mathrm{~mm}^{2}$ of which $33 \%$ are transparent for X-rays

full scale prototype instrument. The operating conditions were: input photon rate up to $2400 \mathrm{c} \mathrm{s}^{-1}$, a ${ }^{60} \mathrm{Co}$ background rate of up to $1000 \mathrm{c} \mathrm{s}^{-1}$ and a temperature range between 0 and $40{ }^{\circ} \mathrm{C}$. The maximum microvibration level was $20 \mathrm{mg}$ in the frequency range between 0 and $1000 \mathrm{~Hz}$.

\subsection{Effective area}

The factors that influence the effective area include:

- the transmission of the thermal foil,

- the mask transparency,

- the beryllium detector window transmission,

- the obscuration by the window support structure,

- the detector efficiency, and

- the instrument lifetime fraction.

The on-axis detector effective area, as derived from a numerical instrument model, is shown in Fig. 8 as a function of incident photon energy. At low energies the efficiency is limited primarily by the transmission of the thermal foil and the beryllium detector entrance window. At high energies the efficiency is limited by the absorption of the gas. The thermal foil consists of a $7.6 \mu \mathrm{m}$ thick kapton filter, aluminium plated by two layers of $0.1 \mu \mathrm{m}$ on each side, the beryllium foil is $150 \mu \mathrm{m}$ thick. The figure does not include the instrument Life Time Fraction. The instrument LTF (This is the inverse of the instrument dead time) is measured once every second during the observations.

\subsection{Energy response}

\subsubsection{Energy to channel conversion}

The energy of an X-ray photon is derived from the main anode (A1) Pulse Height (PH) and digitised in 4096 ADC channels. In the normal operational mode (NM), binning takes place into 31 energy channels. Figure 9 gives the relation between the energy channel boundaries and the photon energy for the two Wide Field Cameras at their nominal detector gain. The width of the channels is chosen such that for an average astronomical spectrum, which 
Table 3. Detector performance characteristics, the optimum quantum efficiency and position and energy resolution is reached at $10 \mathrm{keV}$. All parameters are as foreseen

\begin{tabular}{lll}
\hline Quantum efficiency & & \\
\hline $2 \mathrm{keV}$ & $10 \%$ & \\
$10 \mathrm{keV}$ & $100 \%$ & \\
$30 \mathrm{keV}$ & $40 \%$ & \\
\hline Energy resolution & (collimated) & (full area) \\
\hline $2 \mathrm{keV}$ & $25 \%$ & \\
$6 \mathrm{keV}$ & $16 \%$ & $20 \%$ \\
$22 \mathrm{keV}$ & $19 \%$ & $22 \%$ \\
\hline Position readout & & \\
\hline number of pixels & $768 \times 768$ & \\
nominal pixel size & $0.375 \times 0.375$ & {$\left[\right.$ mm $\left.{ }^{2}\right]$} \\
differential non-lin. $(90 \%)$ & $<0.5$ & {$[$ pixel] } \\
integral non-lin. $(90 \%)$ & $<1.0$ & {$[$ pixel] } \\
\hline Position resolution & $x$-dir [pixel] & $y$-dir [pixel] \\
\hline $2 \mathrm{keV}$ & $<1$ & $<1.5$ \\
$8 \mathrm{keV}$ & $<1$ & $<1$ \\
$22 \mathrm{keV}$ & $<2$ & $<2$ \\
\hline
\end{tabular}

falls exponentially off to higher energies, the counts in each channel are of the same order of magnitude.

The relation between $\mathrm{PH}$ and the corresponding X-ray energy as a function of energy has been calibrated. The calibration has been performed in detail for a small part of the sensitive area, called the Reference Location (RL). The full sensitive area has been calibrated by measuring the gas gain for each pixel with respect to RL, as described in Sect. 3.3.2. The measurements at RL have been performed by means of radioactive sources. Also a fluorescence source with an interchangeable target stimulated by a radioactive alpha emitter was used.

At the nominal gain level the energy - $\mathrm{PH}$ relation is non-linear due to space charge effects in the avalanche. A second order polynomial is least square fitted to the data points up to $8 \mathrm{keV}$ as shown in Fig. 10. The statistical error in $\mathrm{PH}$ is less than $20 \mathrm{eV}$ FWHM. A discontinuity in the energy - $\mathrm{PH}$ relation at the $4.8 \mathrm{KeV} \mathrm{Xe}$ L-absorption edges is included. We found an optimum fit for a discontinuity of $65 \mathrm{eV}$. This value is in good agreement with the value reported by Santos et al. (1991).

\subsubsection{Positional dependence of the gain}

The gas gain of the detector is depending on the position of the photon in the sensitive area. The variation of the gain over the sensitive area was measured by irradiating the full sensitive area. For each irradiated pixel the gain relative to the gain at the Reference Location (RL)

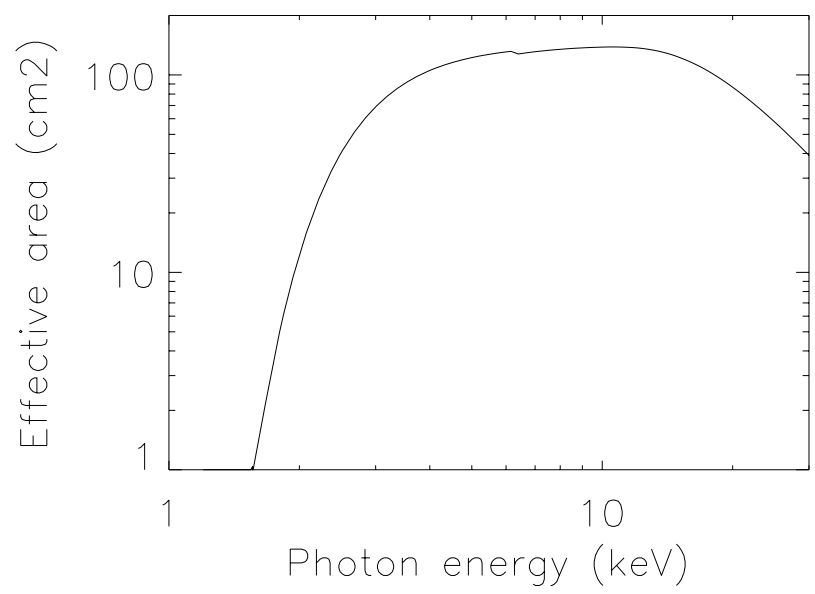

Fig. 8. The total calculated on-axis detector effective area. At low energies the effective area is limited by the thermal foil and detector window. At high energies the efficiency is mainly limited by the stopping power of the detector gas. The maximum effective area is $138 \mathrm{~cm}^{2}$ at $10.5 \mathrm{keV}$

was determined. In Fig. 11 a typical plot is shown of the relative gain as a function of the position along a cross section of the sensitive area in the $y$-direction. This is the direction perpendicular to the anode wires. In the graph open areas indicate the obscuration by the window support structure. The statistical error in the data points is less then $1 \%$ FWHM.

The curve shows two components. A small scale and a large scale gain variation. The small scale component with a modulation length of 8 pixels is caused by the anode wires. The gain maxima coincide with the position in between two anode wires. At those locations the gain is less affected by space charge than at other locations. This is because the cloud of primary electrons is split up between the two anode wires resulting in a lower avalanche space charge density. As the space charge density increases with the photon energy, the modulation amplitude is much larger at $8 \mathrm{kev}(6 \%)$ than it is at $2 \mathrm{keV}(2 \%)$. Small deviations in the positioning of the anode wires result in an additional small scale random modulation of the gain.

The large scale component in the gain variation is almost the same for 2 and $8 \mathrm{keV}$ radiation. The curves show a rather flat distribution. In the last two subwindows the gain is stepwise increased with about $6 \%$. All other cross sections of the sensitive area in the $y$-direction show the same gain step. This feature is probably due to a stepwise decrease of the anode wire diameter with about $0.2 \mu \mathrm{m}$. At both edges of the sensitive area the gain is increasing with about $8 \%$ over a few pixels due to wall effects. 


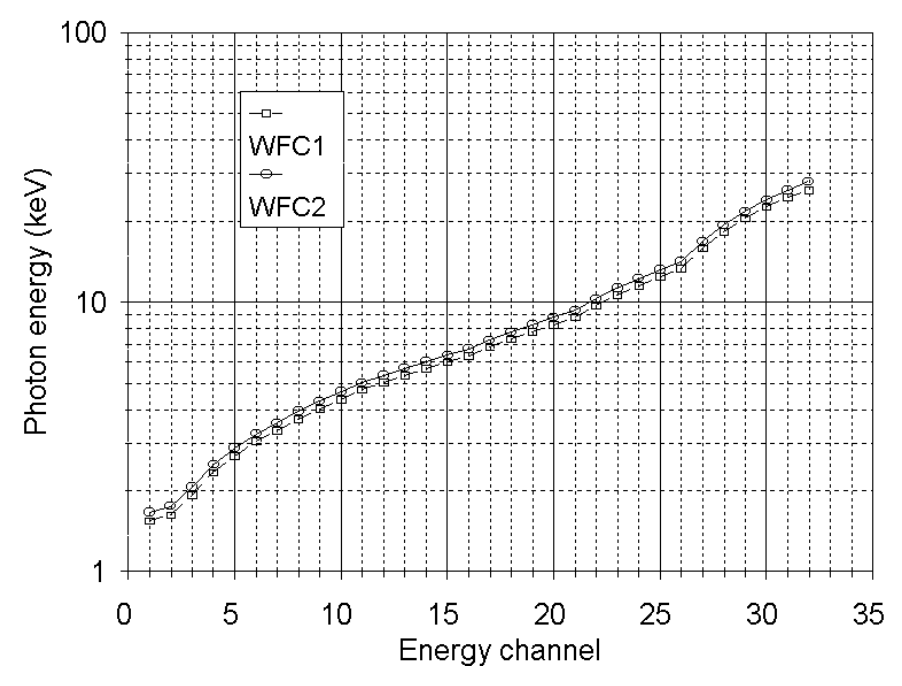

Fig. 9. The relation between the logarithm of the photon energy and the energy bands for the two instruments at nominal gain. The instruments show a small difference due to slightly different gain settings

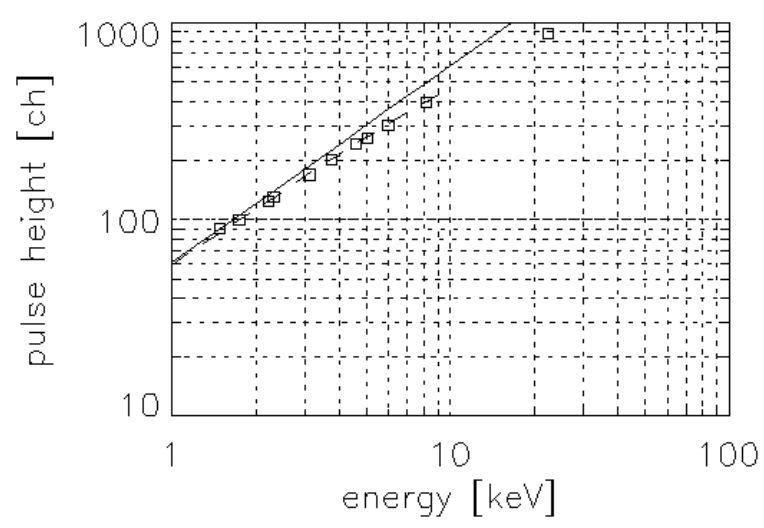

Fig. 10. The detector gain is shown as a function of energy, the response is non-linear at higher energies due to space charge effects in the avalanche. The drawn line is a second order polynomial fit to the data below $8 \mathrm{keV}$

\subsubsection{Energy resolution}

Up to $8 \mathrm{keV}$ the pulse height FWHM (expressed in $\mathrm{keV}$ ) at the Reference Location RL can be described by the relation $0.4 \sqrt{E}$ as shown in Fig. 12. The measured FWHM is in agreement with previous results (Bruel et al. 1988). The resolution at $22 \mathrm{keV}$ however is dominated by the difference in gain between events in the drift region and events in the amplification region and does not follow the relation.

The spectral resolution for full area irradiation is limited by the positional dependence of the gain. At $6 \mathrm{keV}$ the resolution for full area irradiation is $20 \%$ FWHM, while for a collimated beam at RL the resolution is $17 \%$.

\subsubsection{Long term stability}

The long term behaviour of the energy response of the WFC detectors has been monitored with the inflight calibration sources during more then two years since the final integration before launch.

During this period the energy resolution for $6 \mathrm{keV}$ photons has not changed within a few percent. For $22 \mathrm{keV}$ photons the energy resolution degraded from 22.7 to $24.3 \%$, which can be explained by minute changes in the gas composition.

Figure 13 shows the pulse height as a function of time for the two WFC detectors. The statistical error in the data points is less than $0.1 \%$ FWHM. Both detectors show the same trend. It can be seen that in the first 100 days the pulse height increased by about $1 \%$, afterwards the pulse height increased with $0.5 \%$ per 300 days. In the first period a significant part of the gain variations is due to minute movements in the housing walls and the grid system. This is caused by vibration, thermal effects and changes in the ambient pressure during qualification tests. However, the main trend in the gas gain can be explained by minute changes in the gas composition and/or a small drift in the high voltage supply. Anyway, the performance of the instrument with typical integration times of $10^{5} \mathrm{~s}$ is not influenced at all by the measured variation, if necessary the gain can be regulated back to nominal by adjusting the high voltage.

\subsection{Spatial response}

The spatial response has been calibrated by "flat field" measurements and by "pinhole" measurements. A flat field response distribution is used to determine the linearity of the spatial response. For this purpose the bars of the window support structure serve as a reference pattern. A typical image of a flat field measurement at $8 \mathrm{keV}$ is given in Fig. 14. It shows the number of photons counted in the detector pixels. The number of counts is a measure of the differential non-linearity i.e. the relative sizes of the pixels with respect to their nominal size. This information will be used in the image deconvolution process, described in Sect. 4.2.1, to improve the quality.

Likewise, the pinhole measurements will be used to calibrate the numerical model of the detector Point Spread Function (PSF) that is used in the image deconvolution.

\subsubsection{Image linearity}

A typical plot of the integral non-linearity i.e., the difference between the measured pixel position and its nominal position, is shown in Fig. 15 for 2 and $8 \mathrm{keV}$. It is given as a function of the position along a cross section of the sensitive area in the $y$-direction perpendicular to the wires 

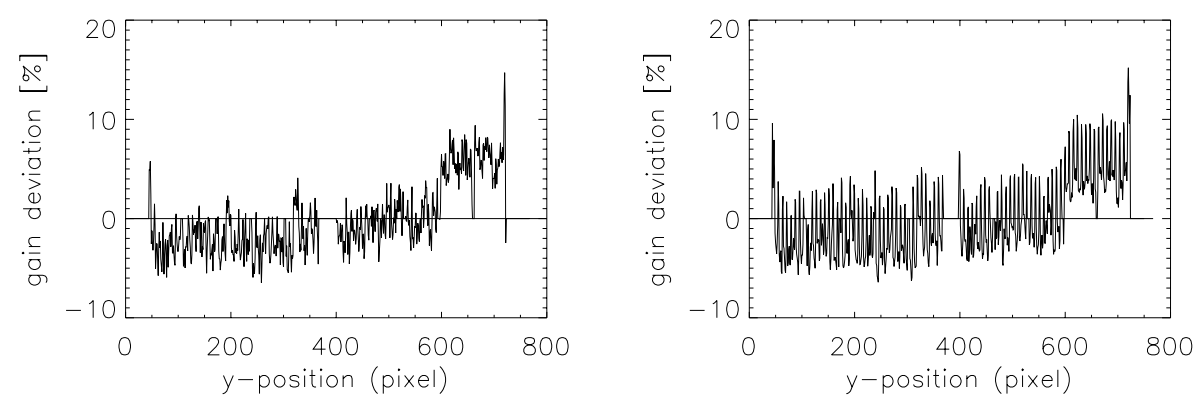

Fig. 11. Positional dependance of the gas gain at $2 \mathrm{keV}$ (left) and at $8 \mathrm{keV}$ (right). The modulation along the $y$-axis is caused by the anode wires and is highest at $8 \mathrm{keV}$ due to the increased space charge density. The step beyond pixel 600 is probably caused by a decrease in anode wire diameter of $0.25 \mu \mathrm{m}$

of the main anode grid A1. The linearity curve has two components: a small scale and a large scale component.

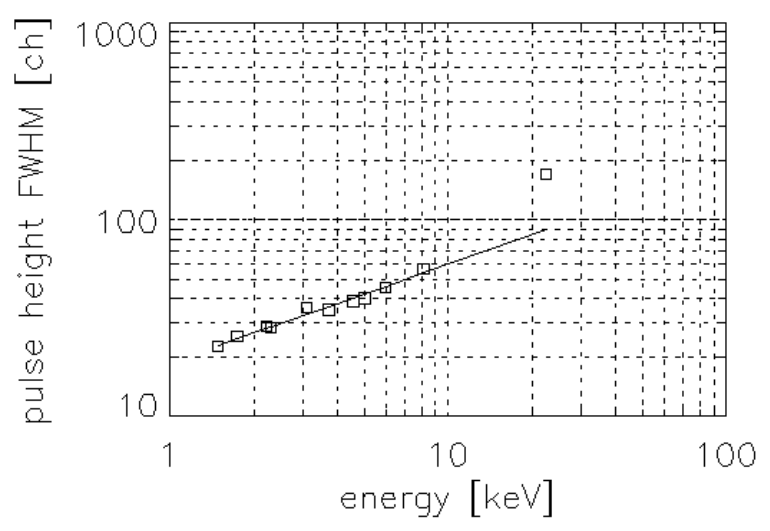

Fig. 12. The energy resolution as a function of energy, up to $8 \mathrm{keV}$ it follows the relation $0.4 \sqrt{E}$

The small scale non-linearity is like the variations in gain caused by the anode wires. The modulation amplitude is the same for 2 and $8 \mathrm{keV}$. The measured small scale non-linearity is the remainder of the total non-linearity caused by the anode wires. A great deal of the latter is already corrected for in the instrument software.

The large scale sawtooth shaped non-linearity component is caused by some bulging of the window support structure and of the window foil in the subwindows. The readout position of the events will be slightly shifted towards the middle of the cushion like deformations because the electric field lines always end perpendicular at the window foil. The modulation amplitude at $8 \mathrm{keV}$ is smaller than at $2 \mathrm{keV}$ due to deeper penetration of the high energy photons. The non-linearity at the first and the last subwindow is larger than at the others. This is because the drift regions are largely but not fully electrostatically shielded from the detector housing walls by field correction plates.

In Fig. 16 a typical histogram of the non-linearity distribution over the pixels is presented for 2 and $8 \mathrm{keV}$. In the $x$ - and $y$-direction more than $90 \%$ of the pixels have a non-linearity of less than 1 pixel which is sufficient for our purpose.

\subsubsection{Point Spread Function}

The spatial resolution for on-axis radiation is better than 1 pixel (FWHM) between 2 and $10 \mathrm{keV}$. In the lower part of the energy range the resolution is limited by the noise of the readout electronics. In the upper part of the range the resolution is limited by the photoelectron track length.

The shape of the spatial response Point Spread Function (PSF) is an important feature for a coded mask camera.

The position and flux of X-ray sources can only be determined by deconvolution (see Sect. 4.2.1, when the PSF is exactly known.

Figure 17 shows a typical plot of the position distribution of events is shown when irradiating the detector through a $0.1 \mathrm{~mm}$ pinhole with $8 \mathrm{keV}$ photons. Because the laboratory background per pixel is very low, the PSF could be measured in great detail. The broad distribution profile under the narrow peak (including the two peaks at both sides of the main peak in the $y$-direction) is caused by events disturbed by pile-up. The level is acceptably low $(\leqq 1 \%)$.

\subsection{Time resolution}

When the WFCs are operated in their imaging mode (Normal Mode), time information is only provided for each block of four detected events. The time resolution is $0.5 \mathrm{~ms}$ and the time tag is valid for the last of the four events. This solution was chosen in order to reduce the number of bits per event, i.e., the telemetry rate. The arrival times of the other three events has to be estimated between this 


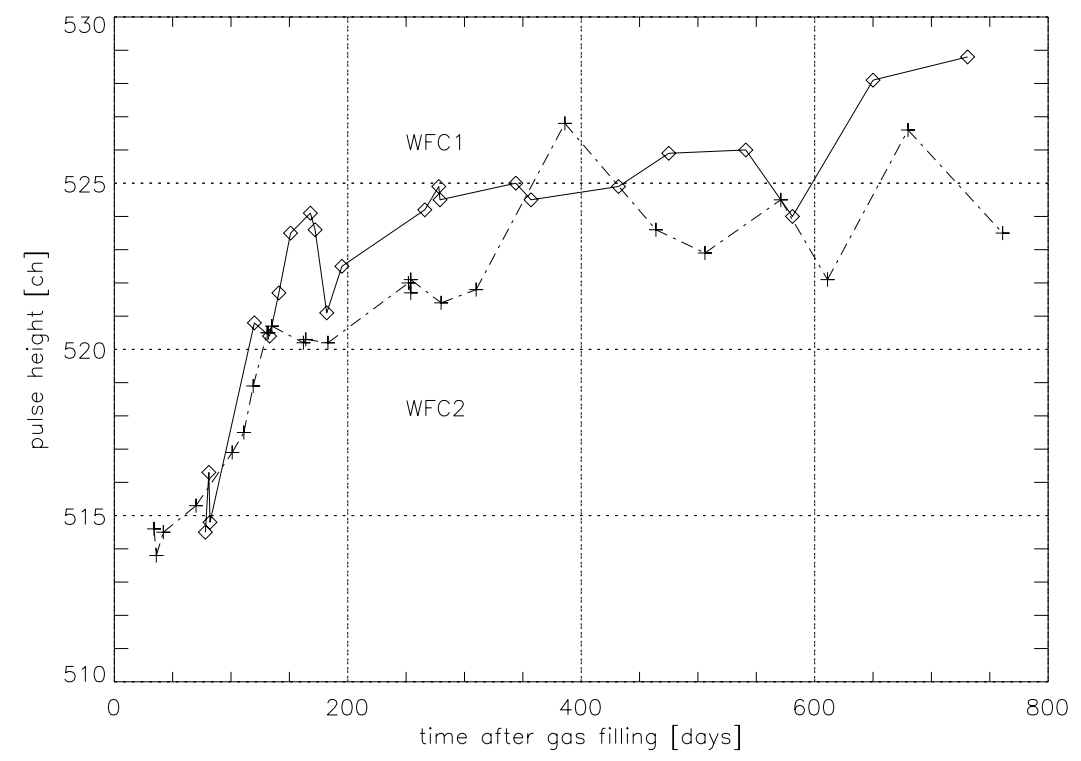

Fig. 13. Long term gain stability of the two WFC flight detectors. After an initial increase, the change in gain has stabilised to less then one percent over a period of one and a half year. The variations are significant and are due to the fact that the instruments were not always completely in thermal equilibrium during the measurements

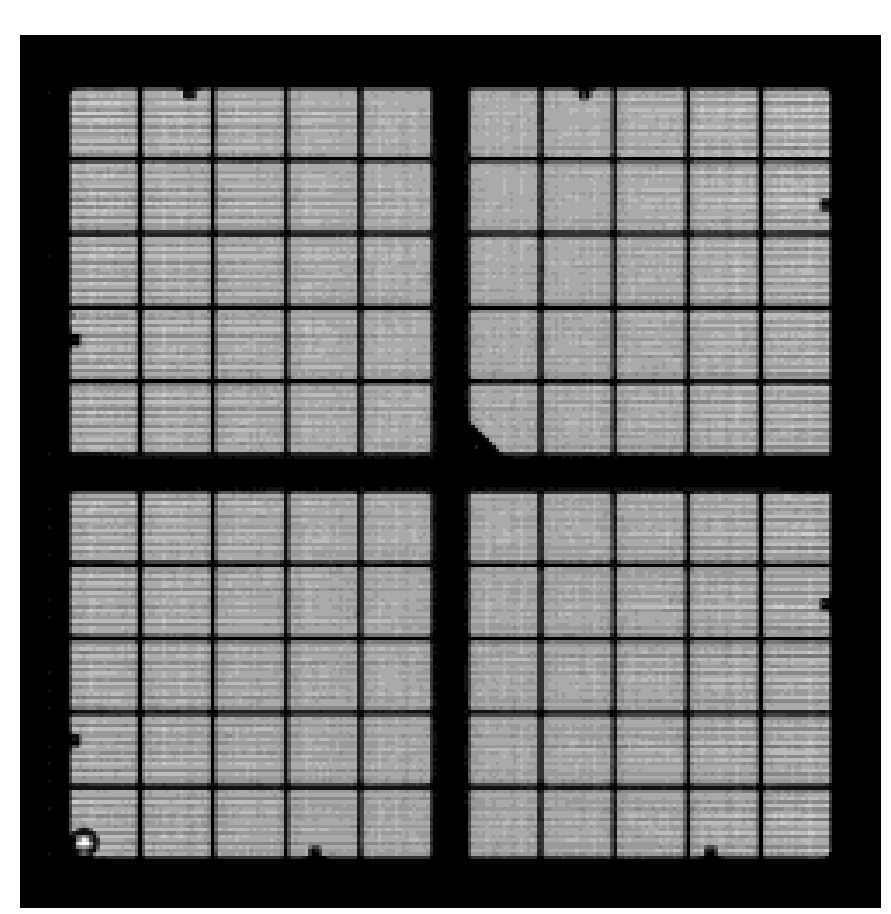

Fig. 14. Flat field measurement performed with $8 \mathrm{keV}$ radiation in the SRON X-ray long beam facility. Clearly visible are the shadow of the detector window support structure, the inflight calibration sources and the modulation by the anode wires which run horizontally time tag and the preceeding one. The accuracy of the time tags is better then $0.08 \mathrm{~ms}$, determined by the uncertainty of certain timing signals on the satellite data bus.

The time resolution in the fast non-imaging mode (High Time Resolution Mode) is $0.25 \mathrm{~ms}$. In this case the time information is provided for each event. Again the precision is better than $0.08 \mathrm{~ms}$.

\subsection{Temperature dependence}

For a number of variables the temperature dependence has been measured over a temperature range of $0^{\circ}$ to $40{ }^{\circ} \mathrm{C}$ (i.e. the nominal operational temperatures). The gas gain varies less than $2 \%$, by means of the inflight calibration sources the actual gain is determined with an accuracy of better than $1 \%$ within 20 minutes. The gain profile over the sensitive area changes less than $0.5 \%$. The position response varies less than 0.2 pixel. Therefore the temperature dependence can be neglected as long as the temperature stays within this temperature range.

\section{Data analysis software}

The telemetry sent by the satellite to the groundstation comprises housekeeping and scientific data. The housekeeping data is used to monitor the overall condition of the satellite and the instruments, and to evaluate the scientific data.

The data from the instruments consists of a list of events labelled with detector position, energy channel and 

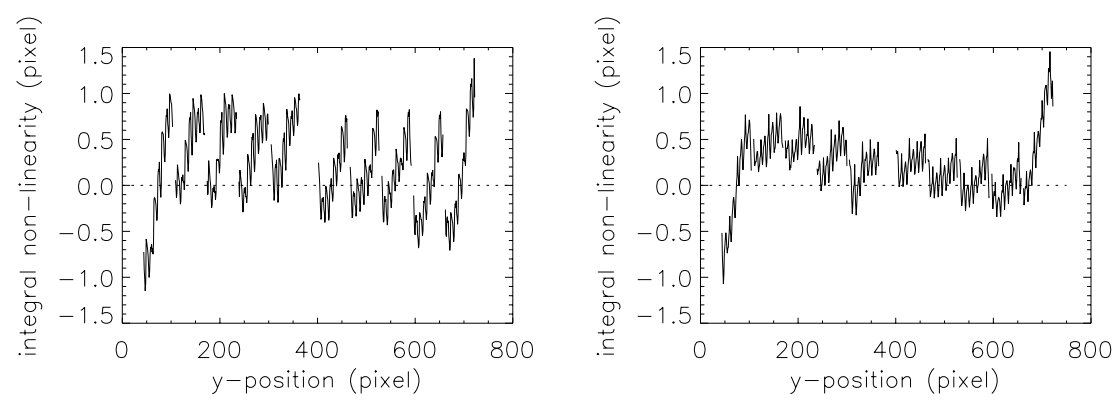

Fig. 15. The integral non-linearity is the difference between the actual and the ideal pixel positions. The left panel displays this effect for $2 \mathrm{keV}$, the right panel for $8 \mathrm{keV}$ photons. The saw-tooth like modulation is caused by the curvature of the Beryllium entrance foil over the subwindows due to the pressure of the gas

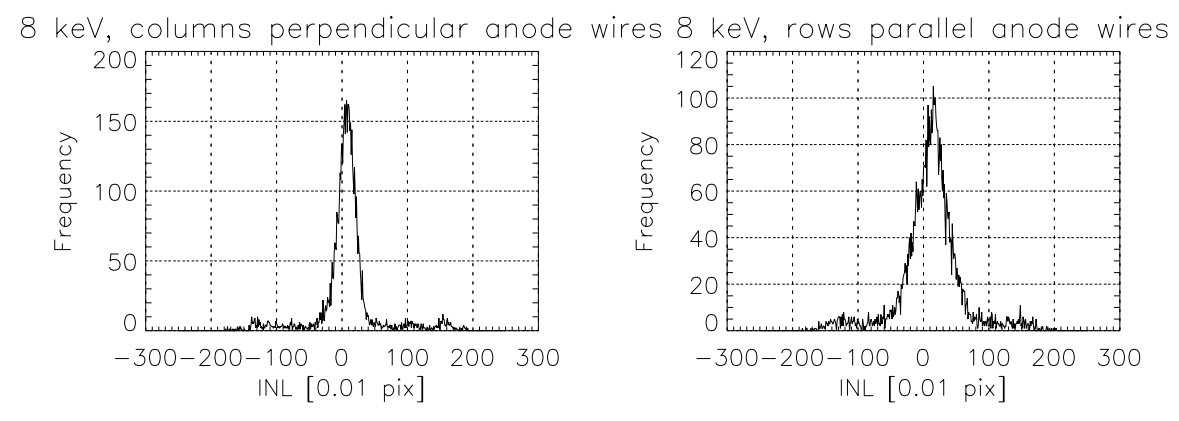

$2 \mathrm{keV}$, columns perpendicular anode wires $2 \mathrm{keV}$, rows parallel anode wires
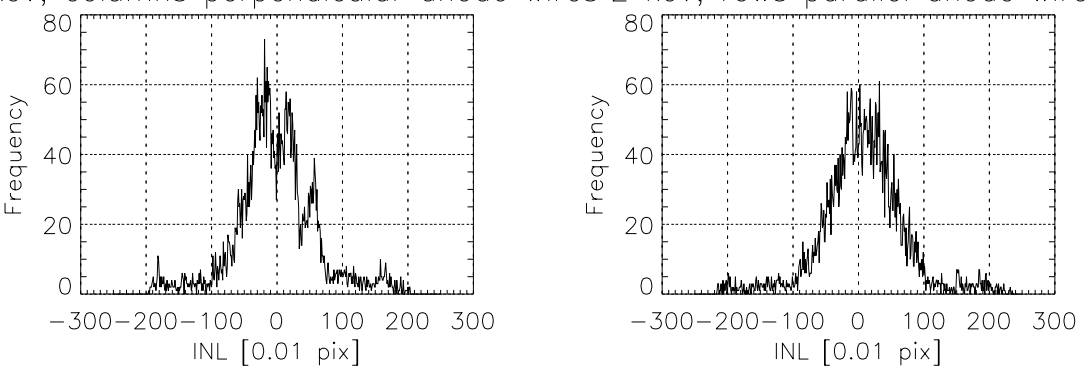

Fig. 16. Histogram of the non-linearity distribution for $2 \mathrm{keV}$ (lower) and $8 \mathrm{keV}$ (upper) illumination and perpendicular (left) and parallel (right) to the anode wires. More than $90 \%$ of the pixels have a non-linearity of less then 1 pixel $(0.375 \mathrm{~mm})$

time of detection. From this raw data one can determine fluxes and positions for all detected celestial X-ray sources (in case of extended sources also morphology). This will all be done on ground.

However, first the instrument and satellite housekeeping must be processed in order to correctly analyse the data. For this purpose software was developed to read the Final Observation Tapes (FOT) and produce cleaned eventlists in FITS format, the so called stage I software. Using these cleaned eventlists, Stage II software creates data products such as images, fluxes, and spectra (Jager et al. 1996).

\subsection{Stage I, FOT processing}

The following extraction and processing activities are carried out on FOTS, in principle without the intervention of the operator:

- Read FOT data from tape or from disk,

- Make WFC specific attitude data, combined with observational mode data and earth occultation information,

- Analyse attitude data in order to produce nominal pointing records reflecting the stability of the pointing or to concatenate datafiles with the same attitudes of different observations, 


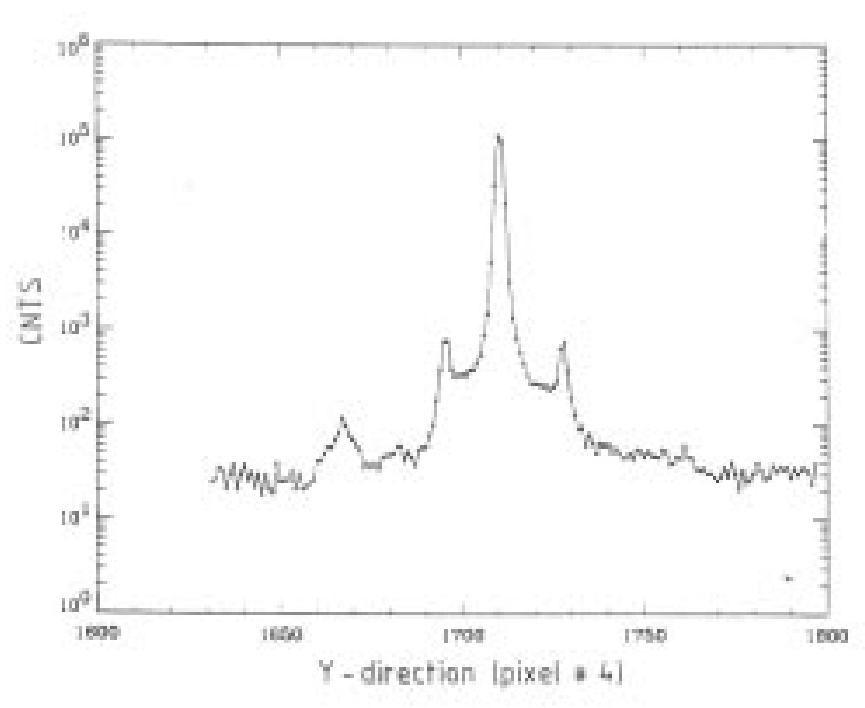

Fig. 17. Point Spread Function in $y$-direction measured at $8 \mathrm{keV}$ through a $0.1 \mathrm{~mm}$ pinhole, the FWHM is 0.6 pixel which is well within the required 1 pixel. The broadened distribution including the two side lobes is acceptably low $(\leqq 1 \%)$, and is caused by pile-up events

- Process WFC housekeeping files and convert all housekeeping values to physical units. Discard records with bad times and produce deadtime fractions. Detect peaks in countrates,

- Create Good Time Interval (GTI) FITS file based on parameters in a housekeeping data FITS file,

- Make plots from WFC housekeeping data and selected other data,

- Filter a FITS table by selecting only the rows that have a time stamp within a specified set of time intervals or that have a ratemeter within a certain range of values,

- Do a bursthunt, flag the existence of a burst for further detailed automatic analysis,

- Merge two or more GTI FITS files, and finally

- Create cleaned event files in FITS format.

\subsection{Stage II, image reconstruction}

The stage I software produces a list of events (photons) that were measured in the detector. Without further analysis, it is impossible to tell whether a photon came from a certain X-ray source, it could have reached the detector through any of the transparent mask elements from almost any part of the sky. Unlike most imaging instruments, the data of a coded mask camera needs first be deconvolved.

The main objective of the stage II software is to reconstruct the observed sky in an optimal time and energy channel interval in order to obtain information on Xray sources. This information typically consists of images, lightcurves and spectra for each source in the field of view. A number of reconstruction methods have been described in the literature, like correlation of the detector image with the mask pattern, the Maximum Entropy Method and Maximum Likelihood Fitting (Willingale et al. 1984). We briefly describe here the method of cross-correlation between detector image and mask pattern. We use this method because it is numerically relatively fast and it has produced satisfactorily results with the COMIS instrument. A full description can be found in in 't Zand (1992). Investigation of other methods is ongoing.

\subsubsection{The correlation method}

Each point source in the large field of view projects the shadow of the mask pattern onto the detector. Extracting the locations and strenghts of these sources from the sum of their projections, means solving an inverse problem. This can be done by deconvolving or cross-correlating the total image on the detector with the mask pattern as follows.

If we represent the detector image of the sky by a twodimensional matrix $D$, then $D$ is given by the convolution $\otimes$ of the mask pattern $M$ and the true sky image $S$ :

$D=S \otimes M$

Reconstruction of the sky image $S^{\prime}$ can be performed by means of a cross-correlation $\odot$ of $D$ with a certain reconstruction matrix $R$, which has to be specified.

$$
\begin{aligned}
S^{\prime} & =D \odot R \\
& =S \otimes(M \odot R) .
\end{aligned}
$$

The reconstruction is perfect if $R$ satisfies the condition

$M \odot R=I$

where $I$ is the identity matrix. If $M$ is based on a pseudorandom URA pattern, $R$ can be found and is uniquely given by $M$. The pattern of $R$ is then equal to the pattern of $M$.

If $D$ contains complete uncorrupted shadows of $M$, Eq. (3) is always exactly matched and $S^{\prime}=S$. However, in a detector-mask configuration such as employed in the BeppoSAX-WFCs (i.e. with equally sized mask and detector, the "simple" camera), $D$ only contains a complete shadow of $M$ for the on-axis position and so-called "coding noise" emerges. The reconstruction of a single sky pixel $k$ can then be represented by

$s_{k}^{\prime}=s_{k}+\sum_{i \neq k} s_{i} \cdot f_{i, k}$

where $s_{k}^{\prime}$ is the reconstructed intensity at sky pixel $k, s_{k}$ the true intensity, and $f_{i, k}$ a factor representing the cross talk between different sky pixels introduced by the coding noise.

Equation (4) illustrates that coding noise is in fact deterministic: if the configuration of sky sources is known, 
the cross talk term may be evaluated and its influence on the estimate of $s_{k}$ eliminated. In practice, suppression of coding noise may be accomplished by an iterative method. Such a method is Iterative Removal Of Sources or IROS (Hammersley 1986; in 't Zand 1992).

In IROS, each step involves the subtraction of simulated point source exposures in the detector plane, where the point sources are those which are significant with respect to the noise in the cross-correlation of that detector image. A cross-correlation on the resulting detector image contains less coding noise and provides the means to evaluate weaker sources for a subsequent iteration. The final product of IROS is a sky image with almost no coding noise and no sources. The subtracted sources can then be displayed in this image.

A problem which arises when using IROS, is the accuracy of the point source location. Due to the coding noise in the image, the Point Spread Functions of the sources are perturbed in a non-statistical manner so that the localisation by means of fitting with the PSF can be slightly off. Subtraction of sources with errors in the positions of only a few tens of a pixel then introduces residues in the image that can not be recovered later. One solution to this problem is to make use of the catalog position of well known sources. After subtraction of the known X-ray sources, any new sources will remain in the sky image and can be analysed. Other possibilities are being studied.

Apart from IROS there are more ways to optimize the signal-to-noise ratio of the reconstruction as determined by the cross-correlation given by Eq. (2), e.g.,

1. Weighing.

The Poisson noise may be suppressed by differently weighing subsections of the detector plane during cross-correlation. The idea behind this technique may be thought of as follows: consider the case when one strong and one weak point source are in the observed sky whose exposures have a small overlap in the detector plane. We may omit counts in this overlap during the reconstruction of the weak source, thus eliminating the strong contribution in Poisson noise from the strong source. This may improve the signal-to-noise of the weak source several tens of percents. Generally, this improvement may be achieved by weighing detector subsections in stead of omission.

2. Transparency correction and detector background subtraction.

The value of the reconstruction matrix $R$ (uniquely given by $M$, see in 't Zand 1992) does not account for possible disturbing influences on the imaging due to the detector window support structure. Part of this disturbance may be eliminated by redefining $R$. The remainder may be eliminated by subtracting an estimate of the contributions by the sky and detector background in the detector plane. This must be done before the cross-correlation. The total countrate of the background was found to be about $190 \mathrm{c} \mathrm{s}^{-1}$ in the full energy passband (cf. the countrate for the Crab X-ray source of $\left.300 \mathrm{c} \mathrm{s}^{-1}\right)$.

The result of the complete reconstruction consists of two images, one representing estimates of the intensity per sky pixel and the other containing estimates of the Poisson noise standard deviation per pixel. Furthermore a list of sources and upper limits is produced for the selected time and energy windows. This can subsequently be used to do a more detailed analysis, i.e. determination of spectra and lightcurves.

\section{Conclusion}

The Wide Field Cameras flying onboard the BeppoSAX satellite represent the latest generation of coded mask $\mathrm{X}$-ray cameras. Their unrivalled field of view, combined with appropriate spatial and temporal resolution (1 arcmin and $0.5 \mathrm{~ms}$ respectively) and modest energy resolution $(20 \%$ at $6 \mathrm{keV})$, make the instruments well suited to perform extensive studies of large areas of the sky and to study transient X-ray phenomena.

Acknowledgements. We want to thank all the people at SRON that have helped us over the years to define and build the Wide Field Cameras. Our special gratitude goes to Dr. R.C. Butler of ASI. Without his unrelenting critical involvement SAX would not have been where it is now.

\section{References}

Bazzano A., Amoretti M., La Padula C., et al., 1994, SPIE 2279, 446

Boella G., Butler R.C., Perola G.C., et al., A\&A (in press)

Boella G., Chiappetti L., Conti G., et al., A\&A (in press)

Bradt H.V.D., Ohashi T., Pounds K.A., 1992, Ann. Rev. A\&A 30, 391

Brinkman A.C., Dam J., Mels W.A., et al., 1983, in Proceedings of the Workshop on Non-thermal and Very High Temperature Phenomena in X-ray Astronomy, Rome, p. 263

Bruel U.G., et al., 1988, SPIE 982, 401

Charpak G., 1985, SPIE 597, 170

Dicke R.H., 1968, ApJ 153, L101

Fenimore E.E., Cannon T.M., 1978, Appl. Opt. 17, 337

Frontera F., Costa E., Dal Fiume D., et al., A\&A (in press)

Hammersley A.P., 1986, Ph.D. thesis, University of Birmingham

Jager R., Heise J., Savenije M.H.F., et al., 1996, ASP Conf. Ser. 101, 240

Manzo G., Giarrusso S., Santangelo A., et al., A\&A (in press) Mels W.A., Lowes P., Buurmans H.B., et al., 1988, Nucl. Inst. Methods A 273, 689

Parmar A.N., Martin D.D.E., Bavdaz M., et al., A\&A (in press)

Pavlinsky M.N., Grebenev S.A., Sunyaev R.A., 1994, ApJ 425, 110

Proctor R.J., Skinner G.K., Willmore A.P., 1979, MNRAS 187, 633

Santos F.P., et al., 1991, Nucl. Inst. Methods A 307, 347 
Sims M., Turner M.J.L., Willingale R., 1980, Space Sci. Inst. Willmore A.P., Skinner G.K., Eyles C.J., et al., 1984, Nucl. 5, 109

Sunyaev R., 1990, Adv. Space Research 10, 233

Willingale R., Sims M., Turner M.J.L., 1984, Nucl. Inst.

Methods P 221, 60 Inst. Methods P 221, 284

in 't Zand J.J.M., 1992, Ph.D. thesis, Space Research Organisation Netherlands, Utrecht

in 't Zand J.J.M., Heise J., Jager R., 1994, A\&A 288, 665 